\title{
Measurement of Excited State Absorption Cross Sections in Dye Solutions with Picosecond Light Pulses
}

\author{
A. Penzkofer and J. Wiedmann
}

Fakultät für Physik. Universität Regensburg, D-8400 Regensburg. Federal Republic of Germany

\section{Introduction}

In excited state absorption measurements intense pump pulses promote molecules to excited states and weak probe beams induce transitions to higher lying states. The measurement of absolute excited state absorption cross-sections is complicated by the fact that the transmission of probe light depends on the number of excited molecules, on their orientational distribution and on the angle between the involved transition moments.

In the past several techniques have been developed.for the measurement of absolute excited singlet state absorption crosssections: $i)$ orientation free absorption spectra were measured with pump and probe beam polarizations oriented under an angle of 54.70 [1]; i i) the excited state absorption was investigated after complete bleaching of the ground state [2]; and $i i i)$ the absorption measurements were compared with numerical simulations [3].

Here a further technique is described which avoids the problems of orientational anisotropy: An intense picosecond pump pulse (frequency $v_{p}$ ) populates the $S_{1}$-state. After molecular reorientation (delay time $t_{D} \simeq 2 \tau_{0}$ ) two probe beams are applied. One probe pulse at frequency. up measures the excited population and the other probe beam at frequency $v_{L}$ monitors the excited state absorption.

The method is applied to determine the absolute excited state absorption cross-sections $\sigma_{e}\left(\nu_{l}\right)$ of rhodamine $6 \mathbf{6}$ and rhodamine $B$ in ethanol for a transition between the singlet states $\mathrm{S}_{1}$ and $\mathrm{S}_{4}$.

\section{Description}

In Fig.l a simplified level scheme of the rhodamine dyes is presented. A pump pulse of frequency $v_{p}=18.960 \mathrm{~cm}^{-1}$ (second harmonic of a modelocked Nd-glass laser; duration $\Delta t_{p}=5 p s$ ) promotes the molecules to the $S_{1}$-band. Excited state absorption 


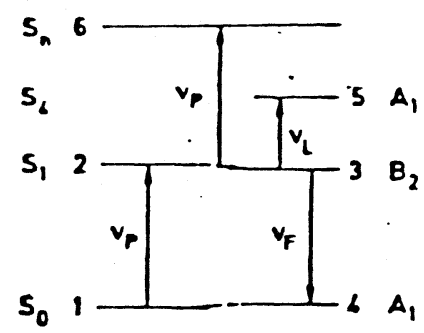

Fio.j lnergy
level diagrami

for rhodamine

dyes.

at frequency $u_{p}$ leads to transitions to level 6 . A probe beam of frequency $v_{L}=9480 \mathrm{~cm}^{-1}$ excites molecules from the $S$ to the $S_{4}$ level. This state is frequentiy termed as $S_{2}$ when two weakly absorbing lower lying singlet states [4] are neglected.

After reorientation of the excited molecules in the $S_{1}$-state the transmission of the probe beam at frequency $v_{L}$ is given by

$$
T_{e}=\exp \left[-\sigma_{e}\left(\dot{v}_{L}\right) \int_{0}^{l} N_{3}(z) d z\right]
$$

$o_{e}\left(v_{L}\right)$ is the isotropic $S_{1}-S_{4}$ excited state absorption crosssection at frequency $v_{L}$.

The total population $\int_{0}^{l} N_{3}(z) d z$ of level 3 is monitored with the probe beam of frequency vp. Its transmission through the sample is

$$
T=\exp \left\{-\sigma_{12} N \ell+\left[\sigma_{12}-\sigma_{e}\left(\nu_{p}\right)\right] \int_{0}^{\ell} N_{3}(z) d z\right\}
$$

$N=N_{1}(z)+N_{3}(z)$ is the total number density of dye molecules in the solution. $\sigma_{1}$ and $\sigma_{e}\left(v_{p}\right)$ are the isotropic ground state and excited state absorption cross-sections at frequency $v_{p}$. $l$ is the sample length.

Solving Eq. 2 for $\int_{1}^{l} N_{3}(z) d z$ and inserting the result into Eq. 1 leads to

$$
\sigma_{e}\left(\nu_{L}\right)=\frac{\ln \left(T_{e}\right)\left[\sigma_{e}\left(\nu_{p}\right)-\sigma_{12}\right]}{\ln (T)+\sigma_{12} N \ell}
$$

Eq: 3 is valid when: i) the excited state population $N_{3}(r)$ is constant over the cross-sections of the probe beams, il) the stimulated emission cross-section $\sigma_{e m}\left(v_{L}\right)$ at frequency $v_{L}$ is negligibly small. iii) the transfer to triplet states may be neglected, iv) the probe beams do not affect the level popula- tions (weak probe beam powers), and $v$ ) an isotropic orientational distribution in the $S_{1}$ state is established (delay. of probe beams $t_{D}=2 \tau_{\text {or }}$ ). 


\section{Experiment}

The experimental setup is depicted in Fig.2. A modelocked Ndglass laser is used. A single pulse is selected with an electrooptical shutter and increased in energy with a Nd-glass amolifier. The second harmonic pump pulse is generated in a KDP crystal. A BKI glass block is used for temporal separation of the fundamental and second harmonic pulses. The transmitted laser pulses at $v_{p}$ and $v_{l}$ are reduced in intensity.and reflected back to the sample. They act as time delayed probe pulses and their transmissions are measured with detectors PDI-4.

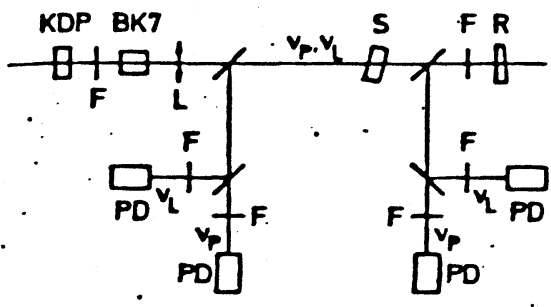

Fig. 2 Experimental arrangement. KDP. crystal for. second harmonic generation; F. filters; BK7, glass block (length $10 \mathrm{~cm}$ ) for temporal separations of probe beams $v_{p}$ and $v_{L}: S$, dye sample; $R$, reflecting glass wedge; $P D$, photodetectors.

\section{4.- Results}

The parameters of the analysed dyes [5]. tonether with the determined excited state absorption cross-sections $\sigma_{e}\left(v_{L}\right)$ are listed in Table 1. The $\sigma_{e}\left(v_{1}\right)$-values are rather small. In case of rhodamine 6G, $\sigma_{e}\left(v_{l}^{-}\right)$shotid be approximately equal to the peak $\mathrm{S}_{1}-\mathrm{S}_{4}$ absorption cross-section (transition to a position slightfy above the $S_{4}$ potential energy curve [6]). The peak. $S^{-S} S_{4}$ absorption in rhodamine $6 G$ is a factor. $g^{f}$ two larger than the $S_{1}-S_{4}$ absorption $\left(\sigma\left(S_{O}-S_{4}\right)=4.5 \times 10^{-17} \mathrm{~cm}^{2}\right.$ at $\left.v=28600 \mathrm{~cm}^{-1}\right)$. Our results agree with Dolan and Goldschmidt [2] who found $\sigma\left(S_{1}-S_{4}\right) \approx 1.5 \times 10^{-17} \mathrm{~cm}^{2}$ for rhodamine $6 \mathrm{~s}$.

The measurement of absolute excited state absorption crosssections at fixed frequencies allows the calibration of qualitative excited state absorption spectra which may be obtained with picosecond light continua [7.].

Table 1

\begin{tabular}{lll}
\hline & Rhodamine $6 \mathrm{G}$ & Rhodamine B \\
\hline $\begin{array}{l}\text { concentration } \\
\text { solvent }\end{array}$ & $1.65 \times 10^{-5} \mathrm{M}$ & $2.9 \times 10^{-5} \mathrm{M}$ \\
$\sigma_{12}$ & ethanol & ethanol \\
$\sigma_{f}\left(v_{\mathrm{P}}\right)$ & $4.17 \times 10^{-16} \mathrm{~cm}^{2}$ & $2.1 \times 10^{-16} \mathrm{~cm}^{2}$ \\
$\sigma_{e}\left(v_{L}\right)$ & $5 \times 10^{-17} \mathrm{~cm}^{2}$ & $5 \times 10^{-17} \mathrm{~cm}^{2}$ \\
\hline
\end{tabular}




\section{References}

1. H.E. Lessing and A. von Jena. Chem. Phys: Lett. 59, 249 (1978).

2. G. Dolan and C.R. Soldschmidt. Chem. Phys. Lett. 39, 320 (1976).

3. A. Mũlier. J. Schulz-Hennig and H. Tashiro. Appl. Phys. 12. $333(1977)$.

4. H. Jacobi and H. Kuhn. Z. Elektrochem. 66. 46 (1962). .

5. W. Falkenstein. A. Penzkofer and W. Kaiser. Ont. Comm. 27. 151 (1978).

6. C. Orner and M.R. Tonp. Chem. Phys. Lett. 36, 295 (1975).

7. A. Penzkofer and W. Käiser. Odt. Yuant. Electr. 9. 315 (1977). 